\title{
MARCADORES TEXTUAIS DE TEXTOS ESPECIALIZADOS EM TRADUÇÃO
}

\section{Viviane Possamai*}

RESUMO: O presente artigo é resultado da investigação de mestrado intitulada Marcadores textuais do artigo científico em comparação português e inglês - um estudo sob a perspectiva da tradução. No referido trabalho, faz-se um estudo de artigos científicos da área de Ciência da Computação, em especial de expressões típicas de seu desenvolvimento e organização, denominadas "marcadores textuais". Após a identificação de tais marcadores, e de seus padrões de uso e colocação em textos em português e em inglês, utilizando-se dois corpora, as mesmas foram analisadas e classificadas com base nas metafunções da linguagem propostas por Halliday. Após essa classificação, comparamos as ocorrências das unidades em inglês e português, observando padrões de uso, freqüência e colocação, no intuito de avaliar diferenças de uso e freqüência que pudessem auxiliar o tradutor na produção de um texto de chegada claro e com menos interferências da lingua de partida. O trabalho visa também a chamar a atenção para unidades típicas de textos especializados diferentes dos termos técnicos, e que, por constituírem o texto especializado, são passiveis de análise por estudos de Terminologia que buscam uma integração com áreas como a de Tradução.

UNITERMOS: marcadores textuais; tradução; retórica contrastiva.

ABSTRACT: This paper is a result of a Master's investigation entitled Textual markers in research articles under the perspective of translation. The thesis focused on scientific articles from the Computer Science area, more specifically on typical expres-

* Doutoranda. Universidade Federal do Rio Grande do Sul. Programa de Pós-Gradução em Letras. 
sions related to their textual development and organization, the textual markers. After the identification of such units, and of their usage and collocation patterns in texts in Portuguese and English, they were analyzed and classified based on Halliday's metafunctions. The objective of the comparison was to observe usage, frequency and collocation patterns, aiming at assessing differences between the two languages in order to provide translators with a support to write Portuguese to English texts with the least of interference from their source language. We are also highlighting how important it is to study components of specialized texts other than the terms when translation is intended to benefit from Terminology studies.

KEYWORDS: textual markers; translation; contrastive rhetoric.

\section{Introdução}

"O tradutor deve saber decodificar as convenções próprias do gênero a que pertence o texto original e saber utilizar as que são próprias do gênero na língua e cultura de chegada, quando essa for a finalidade da tradução".

Hurtado Albir (2001: 491)

Muito se acredita que na tradução técnica o detentor de terminologia de uma dada área é obrigatoriamente melhor tradutor do que quem não a detém (Benedetti, 2003). Aspectos discursivos de um texto técnico ou científico, ou seja, outros elementos que fazem parte das linguagens especializadas e que estão no mesmo ambiente que o termo são por vezes esquecidos por parte de quem tem no texto especializado e na tradução seus objetos de estudo e trabalho.

Sabe-se que certos tipos de textos mantêm um forte grau de convenções, lingüísticas e pragmáticas, que os restringem e moldam. As expressões que enfocamos neste trabalho - marcadores textuais - fazem parte dessas convenções; elas estruturam, organizam e dão o caráter retórico do gênero textual em questão: o artigo científico.

TradTERM, 12, 2006, p. 159-179 
$\mathrm{Na}$ elaboração de um artigo, fazem-se presentes muitos fatores que condicionam a sua produção, pertencentes ao contexto da comunidade científica e acadêmica à qual o texto pertence, incluindo colegas, adversários, editoras, instituições, o histórico da área e todo o entorno de uma pesquisa. O reflexo dessas influências pode ser percebido tanto nos padrões de texto como nas expressões utilizadas.

Tendo em vista que os artigos científicos são aceitos e reconhecidos como constituintes da comunicação de uma grande comunidade, não apenas a de cada língua, mas também a da grande comunidade científica internacional, e que seu formato é já bastante estável (a divisão nas seções "Introdução", "Métodos", "Resultados" e "Discussão", por exemplo), considera-se de fundamental importância que correspondam ao que essas comunidades esperam deles, tanto em português quanto em inglês, como realizações de um gênero específico.

Nesse cenário, entendemos que, embora o artigo científico seja já identificado como um gênero estável, ainda é necessário, para a tradução, identificar, no nível textual, as estruturas que o compõem e o fazem acontecer como um gênero sob um ponto de vista contrastivo entre os idiomas envolvidos. As estruturas que foram foco deste trabalho fazem parte dessas regularidades, e o objetivo principal da pesquisa foi identificar tais estruturas para posterior análise de semelhanças e diferenças em textos em inglês e em português. Com isso, visou-se a encontrar uma forma de auxiliar a realização de traduções (no sentido português-inglês) que se encaixem nos padrões textuais esperados das culturas e das comunidades em que se realizam. Tal ponto de vista encontra suporte nos estudos da Retórica Contrastiva e nos conceitos de gênero desenvolvidos por Mikhail Bakhtin (1997) e J. Swales (1990), como discutiremos na seção 4. Foram utilizadas as ferramentas empregadas pela Lingüística de Corpus para compilação e análise dos textos e o modelo sistêmico-funcional de Halliday (1985) para a classificação das unidades identificadas.

O artigo está estruturado da seguinte forma: a seção 2 descreve o corpus e a metodologia de pesquisa, a seção 3 apresenta os resultados da análise do corpus e a classificação das 
unidades identificadas, além de algumas considerações a respeito da análise comparativa entre as duas linguas. As seções 4 e 5, respectivamente, apresentam a discussão e as considerações finais.

\section{Descrição do corpus e metodologia}

As unidades que são objeto de investigação deste trabalho foram extraídas de um corpus de artigos científicos em português e de outro composto de artigos científicos em inglês. Utilizamos pressupostos e ferramental da Lingüistica de Corpus (LC) para o manuseio, preparação e escolha dos textos (BerberSardinha, 2004). O software utilizado foi o Wordsmith, em especial as ferramentas WordList e Concordance. Podemos então dizer que esta é uma pesquisa baseada em corpus, que utilizou suas ferramentas para comprovação de um pressuposto estabelecido com base em observações dos textos, qual seja, de que as unidades marcadoras do texto de artigos científicos em cada um dos idiomas estudados, inglês e português, apresentam diferenças e que, na maioria das situações de tradução de textos para o inglês, há uma interferência do modo de dizer e escrever do português no texto em inglês. O objetivo do levantamento das unidades nos corpora procurou ser extensivo e identificar o máximo possível de unidades.

\subsection{Descrição dos corpora}

O corpus em português é constituído por 333 artigos científicos que totalizam 931.719 palavras. Os textos foram coletados dos Anais dos Congressos da Sociedade Brasileira de Computação de 2001, 2002 e 2003, que congregam uma série de seminários, conforme mostra a Tabela 1 . 
Tabela 1 - Composição do corpus em português. Número de textos de cada ano por seminário.

\begin{tabular}{lrrrrr}
\hline & 2001 & $\mathbf{2 0 0 2}$ & $\mathbf{2 0 0 3}$ & $\begin{array}{c}\text { TOTAL } \\
\text { (Textos) }\end{array}$ & $\begin{array}{c}\text { TOTAL } \\
\text { (Palavras) }\end{array}$ \\
\hline SEMISH & 7 & 10 & 8 & 25 & \\
WEI & 14 & 11 & 11 & 36 & \\
WIE & 34 & 65 & 60 & 159 & \\
ENIA & 44 & - & 50 & 94 & \\
WPerformance & - & 11 & 8 & 19 & \\
\hline & & & & & 333 \\
\hline Total (textos) & 99 & 97 & 137 & & $\mathbf{9 3 1 . 7 1 9}$ \\
\hline Total (palavras) & & & & & \\
\hline
\end{tabular}

Semish = Seminário Integrado de Software e Hardware; WEI = Workshop sobre Educação em Computação, WIE = Workshop de Informática na Escola, ENIA = Encontro Nacional de Inteligência Artificial, WPerformance = Workshop em Desempenho de Sistemas Computacionais e de Comunicação.

O corpus em inglês constitui-se de 111 artigos, que totalizaram 872.182 palavras e, diferentemente dos textos que constituíram o corpus em português, foram extraídos de diferentes fontes. Como não encontramos anais de um evento semelhante ao da SBC, com tanta diversidade, optamos por procurar artigos que fossem de áreas semelhantes às dos textos em português. Assim sendo, utilizamos recursos da internet para fazer a busca dos artigos. Uma das fontes utilizadas foi o Portal de Periódicos da Coordenação de Aperfeiçoamento e Pesquisa em Ensino Superior (CAPES), cujo acesso é disponibilizado às Instituições de Ensino Superior (IES). Através do portal, chegamos às publicações das revistas Artificial Intelligence e Robotics and Autonomous Systems; para os textos na área de informática na educação, educação à distância e engenharia de software, utilizamos o site de busca Cite Seer e o site dos periódicos eletrônicos Canadian Journal of Distance Education (CADE) e Canadian Journal of Learning and Technology (CJLT) (Ver detalhamento na Tabela 2).

Cuidamos para que todos os textos tivessem sido escritos em universidades de países cuja língua nacional fosse o inglês. Como não era possível verificar a nacionalidade de cada autor, utilizamos esse critério da instituição para garantir que o texto 
havia sido escrito, no mínimo, em um contexto de lingua inglesa, sendo assim muito provável que os textos não houvessem sido traduzidos, mas produzidos diretamente em inglês.

Tabela 2 - Composição do corpus em inglês. Número de textos por ano de acordo com periódico.

\begin{tabular}{|c|c|c|c|c|c|}
\hline & 2002 & 2003 & 2004 & $\begin{array}{l}\text { TOTAL } \\
\text { (textos) }\end{array}$ & $\begin{array}{c}\text { TOTAL } \\
\text { (palavras) }\end{array}$ \\
\hline Artificial Intelligence & & 17 & 3 & 20 & \\
\hline $\begin{array}{l}\text { Canadian Journal of Distance } \\
\text { Education }\end{array}$ & 7 & 3 & - & 10 & \\
\hline $\begin{array}{l}\text { Canadian Journal of Learning } \\
\text { and Technology }\end{array}$ & 16 & 8 & - & 24 & \\
\hline $\begin{array}{l}\text { Robotics and Autonomous } \\
\text { Systems }\end{array}$ & - & 22 & - & 22 & \\
\hline $\begin{array}{l}\text { CiteSeer /Informática na } \\
\text { educação }\end{array}$ & - & - & 15 & 15 & \\
\hline $\begin{array}{l}\text { CiteSeer/Software } \\
\text { Development }\end{array}$ & - & - & 20 & 20 & \\
\hline
\end{tabular}

\begin{tabular}{lccccc}
\hline Total (textos) & 23 & 50 & 38 & 111 & \\
\hline Total (palavras) & & & & & 872.182 \\
\hline
\end{tabular}

O corpus em inglês constitui-se de um número três vezes menor de textos do que o corpus em português; no entanto, observa-se que os artigos selecionados para o corpus em inglês são um pouco mais extensos e a diferença no número de palavras não é significativa. Ressalta-se ainda que, não fosse pelas ferramentas utilizadas pela LC, não seria possivel lidar com tamanha quantidade de textos.

\subsection{Levantamento e identificação dos marcadores discursivos}

Para que conseguíssemos apreender as ocorrências das unidades que interessavam à pesquisa e comprovar que elas seriam típicas do gênero em questão, foi preciso uma abordagem de duas vias, quantitativa e qualitativa, que não foram aplicadas em separado. O critério quantitativo foi utilizado nos dois momentos de análise da pesquisa, tanto estabelecendo o núme- 
ro mínimo de ocorrências que uma determinada expressão deveria ter para constituir-se como típica, no corpus em português, como após o levantamento, servindo a freqüência como critério de avaliação de preferência de uso em cada uma das línguas. O critério qualitativo foi utilizado, juntamente com o quantitativo, para selecionar as unidades que fariam parte da análise, uma vez que, ao realizarmos uma lista de freqüência de agrupamentos (clusters), diversas unidades eram retornadas, não apenas as que interessavam para a pesquisa (ex.: nomes de universidades).

Trabalhou-se primeiramente com o corpus em português. O primeiro passo dado foi a realização de uma lista de freqüência dos clusters de quatro palavras, ou seja, em todo o corpus, quantas vezes uma determinada seqüência de quatro palavras se repetia. Para essa tarefa, foi utilizada a ferramenta WordList, do software Wordsmith Tools. O objetivo foi encontrar resultados que nos levassem à apreensão dos marcadores textuais típicos do artigo científico, através do critério de freqüência de realização. A idéia foi de não utilizar o corpus para buscar unidades que já conhecíamos como típicas dos artigos, mas deixar que os textos demonstrassem por si as unidades que lhe eram particulares. Essa primeira ação resultou em 29.554 ocorrências, com freqüência de repetição dos clusters de 148 a 2. Apresentamos, a seguir, os 10 primeiros agrupamentos, a título de exemplificação:

$\begin{array}{lll}\text { N } & \text { Word } & \text { Freq. } \\ 1 & \text { de ciência da computação } & 148 \\ 2 & \text { de acordo com a } & 137 \\ 3 & \text { em sala de aula } & 135 \\ 4 & \text { com o objetivo de } & 118 \\ 5 & \text { para o desenvolvimento de } & 104 \\ 6 & \text { de acordo com o } & 103 \\ 7 & \text { de informática na educação } & 102 \\ 8 & \text { do ponto de vista } & 102 \\ 9 & \text { de sistemas de informação } & 100 \\ 10 & \text { de computação e informática } & 87\end{array}$


Analisamos os agrupamentos que tiveram de 148 a 5 ocorrências em todo o corpus, o que totaliza 3.449 ocorrências. Dessas, nem todas satisfaziam o interesse da pesquisa; por exemplo, os nomes das universidades que estavam no cabeçalho dos artigos. Foram, então, selecionadas cerca de 400 ocorrências que consideramos poderiam ser parte de ou serem as próprias unidades a serem estudadas. Essa seleção foi feita qualitativamente, empregando o conhecimento que tínhamos do gênero de texto em questão. Utilizando os exemplos da lista acima apresentada, selecionamos as sublinhadas:

$\begin{array}{lll}\text { N } & \text { Word } & \text { Freq. } \\ 1 & \text { de ciência da computação } & 148 \\ 2 & \text { de acordo com a } & 137 \\ 3 & \text { em sala de aula } & 135 \\ 4 & \text { com o objetivo de } & 118 \\ 5 & \text { para o desenvolvimento de } & 104 \\ 6 & \text { de acordo com o } & 103 \\ 7 & \text { de informática na educação } & 102 \\ 8 & \text { do ponto de vista } & 102 \\ 9 & \text { de sistemas de informação } & 100 \\ 10 & \text { de computação e informática } & 87\end{array}$

Dessas cerca de 400 ocorrências, verificou-se de que maneira cada uma ocorria nos textos, quais os verbos que as acompanhavam, se estavam em início ou fim de frase, se realizavam a mesma função, se apresentavam variações de singular e plural, masculino e feminino, se os verbos eram conjugados. Por exemplo, para a expressão "de acordo com a", verificamos se era sempre seguida do artigo feminino, se não, quais eram os artigos, definidos ou indefinidos, no singular ou no plural, que ocorriam com essa expressão e, se não fosse seguida de artigo, que outro elemento a constituía. Para a expressão "a figura $x$ apresenta", verificávamos se ocorria em outros tempos verbais, ou com outros verbos.

Para essa tarefa, utilizamos a ferramenta Concord do Wordsmith Tools e passamos em revista uma a uma as 400 ocorrências, pois sabiamos também que poderiam existir expressões com mais do que as quatro palavras definidas para iniciar a 
pesquisa (clusters de quatro palavras). Com a ferramenta Concord, foi possivel resgatar todas as ocorrências de uma palavra ou expressão no corpus, verificando suas colocações e freqüências.

As ocorrências foram então sendo agrupadas de acordo com semelhanças na função que desempenhavam no texto, por conterem a mesma palavra-chave ou por semelhanças sintáticas. Após esse agrupamento por função ou palavra-chave, percebeu-se que podiamos sistematizar a classificação ainda mais, e aî chegamos à classificação em três grandes grupos, que posteriormente encontraram apoio do modelo sistêmico-funcional que demonstraremos a seguir na discussão. Satisfatoriamente, a classificação empírica realizada através da análise do corpus entrava em conformidade com esses pressupostos postulados por Halliday sobre as macrofunções da linguagem. As três grandes categorias identificadas foram marcadores relacionados ao propósito do artigo científico, marcadores metarreferenciais e marcadores subjetivos, não esquecendo que, dentro de cada uma das categorias, os marcadores foram agrupados por palavranúcleo, ou palavra-chave, como se verá na Seção 3.

Por fim, calculou-se um percentual da ocorrência desses marcadores nos textos. Assim, por exemplo, a expressão a figura $x$ apresenta teve um percentual de 22,2\%. Esse percentual é de quantas vezes a expressão apareceu no total de 333 textos. Por esse motivo, às vezes o percentual ultrapassa os $100 \%$, significando que tal expressão ocorreu mais de uma vez em um mesmo texto.

Com os textos em inglês, realizou-se procedimento semelhante. Foi feita a lista de freqüência de agrupamentos de quatro palavras, utilizando-se a ferramenta WordList. Em inglês, tivemos um resultado de 34.081 agrupamentos, que foram de uma freqüência de 489 a 2. Dessas, selecionamos as que tinham ocorrência de até cinco vezes em todo o corpus, o que resultou um total de 2.215 ocorrências.

Fez-se a mesma avaliação qualitativa, selecionando-se as unidades que demonstravam ser organizadoras, descritoras e típicas do texto do artigo cientifico. Estas foram também analisadas com auxílio da ferramenta Concord, e agrupadas confor- 
me as três categorias que se estabeleceram para as unidades em português (marcadores relacionados ao propósito do artigo cientifico, marcadores metarreferenciais e marcadores subjetivos).

As freqüências de uso foram calculadas para efeito de comparação com as unidades em português.

Criamos, então, quadros comparativos ${ }^{1}$ em que foram observadas semelhanças e diferenças de uso e freqüência dessas expressões em inglês e português, algumas das quais serão mencionadas na seção Resultados, a seguir.

\section{Resultados}

Nesta seção, apresentamos alguns exemplos da análise comparativa que fizemos dos marcadores textuais, mostrando uma equiparação de seus usos e freqüências em português e inglês, por meio de alguns exemplos. É preciso mencionar que a comparação realizada visa, em primeiro lugar, a instrumentar a reflexão por parte do tradutor, ou de quem escreve um artigo científico em inglês, sobre como irá traduzir/utilizar os marcadores no texto, baseando-se nos resultados apresentados no levantamento feito com os corpora.

Os resultados foram sistematizados, no texto completo da dissertação, na forma de quadros, em que foi possivel visualizar de maneira mais clara os marcadores identificados e sua freqüência de uso em inglês e português. Como o resultado do levantamento mostrou um grande número de expressões, selecionamos, para comentário, apenas alguns marcadores.

Conforme mencionado, o elenco de marcadores levantado com o corpus ficou assim sistematizado:

\section{- Marcadores relacionados ao propósito do artigo cien- tifico}

\footnotetext{
1 Por motivos de limitação de espaço, os quadros não foram apresentados neste artigo. Para referência completa, ver Possamai, V. (2004). Marcadores textuais do artigo científico em comparação português e inglês - um estudo sob a perspectiva da tradução. 165 f. Dissertação de Mestrado. UFRGS.
} 
Esses marcadores relacionam-se a elementos do propósito do artigo científico, cujo texto visa, de maneira geral, a apresentar objetivos e objetos do estudo, descrever a fundamentação teórica, ações e métodos e apresentar conclusões. Foram agrupados por palavras-núcleo e grupos funcionais, como mostram os exemplos a seguir.

Agrupados por palavra-núcleo: "objetivo" e "forma" (purpose e way)

Exemplos:

Português: "com o objetivo de", "o objetivo central deste estudo", "da mesma forma que", "é uma forma de"

Inglês: "for the purpose(s) of", "the purpose of this study", "in the same way as"

Agrupados por grupos funcionais: método, ancoragem, quantificação, tempo, explicação e restrição.

Exemplos:

Português: "com o auxílio de", "a partir da análise", "na maioria das vezes", "até o presente momento", "como por exemplo", "no que diz respeito a"

Inglês: "with the help of", "serves as a basis for", "for the most part", "over a period of", "on the one hand ... on the other", "with respect to the"

\section{- Marcadores metarreferenciais}

Os marcadores textuais desse grupo dizem respeito à organização formal e à ordenação do texto do artigo científico e dos elementos que fazem parte dele: seções, figuras, tabelas.

Esses marcadores foram divididos em sete grupos em torno das palavras-núcleo "seção", "figura", "tabela", "artigo", "trabalho" e de um grupo específico de estruturas que se referem explicitamente à organização do texto.

Agrupados por palavra-núcleo: seção, figura, tabela, artigo, trabalho (figure, table, paper)

Exemplos: 
Português: "a seção (número) apresenta", "como mostrado na figura", "conforme pode ser visto na tabela", "este artigo apresenta", "este trabalho descreve"

Inglês: "section (número) presents", "as illustrated in figure", "is shown in table", "this paper describes"

Agrupados pelo grupo funcional de organização:

Português: "o artigo está organizado da seguinte forma", "este artigo está organizado da seguinte forma"

Inglês: "the paper is organized as follows", "in the reminder of this paper"

\section{- Marcadores subjetivos}

Foram assim denominados porque trazem consigo, e deixam transparecer, a visão do sujeito que realiza o artigo. Embora esses marcadores tenham essa característica que os distingue dos mais tipicamente textuais, estão presentes nos corpora e são indissociáveis da constituição de um artigo científico. Contam com a presença de modalizadores e foram divididos em cinco subgrupos, de acordo com as seguintes palavras-núcleo: "importante", "necessário", "esperar"/"acreditar", "observar", "poder".

Agrupados por palavra-núcleo: "importante", "necessário", "esperar" /"acreditar", "observar", "poder" (important, necessary, believe, notice, say)

Exemplos:

Português: "é importante ressaltar que", "é necessário que se", "espera-se que", "pode-se perceber que", "pode ser visto(a) como" Inglês: "it is important to note that", "it is necessary to", "we believe that", "notice that", "we will say that"

Cada um dos marcadores em português, e a sua comparação em inglês, seria merecedor de um estudo que pudesse avançar ainda mais, descrevendo, detalhadamente, as formas de realização de cada um deles em cada uma das línguas. Este trabalho, no entanto, apenas traz os exemplos acima mencionados e tece alguns comentários sobre o resultado do levantamento, de caráter mais genérico. 
Sobre as unidades em português, foi possivel observar que:

- os marcadores textuais relacionados ao propósito do artigo científico mostraram ter predominantemente a forma de locuções, com a presença de preposições, como "a fim de", "de forma que", "a partir de", "com o uso de";

- os marcadores metarreferenciais mostraram-se como formas mais fraseológicas, compostos de uma palavra-núcleo ("tabela", "figura", "seção") e verbo: "a seção $x$ apresenta", "conforme descrito na seção $y$ ”, "como ilustra a figura $z$ ", "este artigo apresenta";

- os marcadores subjetivos mostraram ter uma forma que introduz orações subordinadas: "é importante ressaltar que", "é necessário que", "esperamos que”, "observa-se que”. A eles, geralmente, está relacionada a presença de modalidades.

Além desses dados genéricos sobre os modos de constituição das estruturas analisadas em português, a comparação entre as linguas nos mostrou que:

- no grupo dos marcadores relacionados ao propósito, a língua inglesa costuma ser mais direta na formulação das expressões; assim, em português, teriamos: "através da realização de um estudo", e, em inglês, teríamos, "by studying" (estudando-se), ou "through the study" (através do estudo);

- a língua inglesa realiza mais construções sintáticas de frases que utilizam ou favoreçam o uso do sujeito na primeira pessoa do plural we (nós). Por exemplo:

(a) In this paper we present a new algorithm for DTP solving, called Epilitis.

(Neste artigo apresentamos um novo algoritmo para a solução de DTP, ...)

(a') Este artigo apresenta a proposta de uma estrutura baseada em agentes inteligentes para a definição de uma seqüência.

- a lingua portuguesa utiliza algumas formas passivas inexistentes no corpus em inglês:

(b) * (nenhuma ocorrência no corpus em inglês)

(b’) Na seção y são apresentados 
O exemplo acima mostra um caso em que, em inglês, a forma mais freqüente favoreceria o uso do sujeito na primeira pessoa, como "in section $y$ we show".

- a língua inglesa apresenta maior freqüência de uso de expressões que organizam explicitamente a estrutura do texto:

(c) This paper is organized as follows

(Este artigo é organizado da seguinte forma)

(c') O artigo está organizado da seguinte forma/como segue

- outra evidência, talvez de menos relevância, é que o inglês utiliza, para fazer referência a elementos como figuras e tabelas, a forma entre parênteses, como em "(see Table 1)", e em português essa forma não mostrou nenhuma freqüência. Apesar de essa evidência parecer de pouca relevância, é um recurso que, no momento da tradução, pode possibilitar que o tradutor faça uma escolha por uma frase mais direta, em inglês, apenas indicando, entre parênteses, onde determinada informação pode ser encontrada; isso em vez de traduzir literalmente uma expressão do português do tipo "como mostra a figura $x$ ".

Essa comparação feita entre as línguas, rapidamente demonstrada aqui, mostra que os textos dos dois sistemas de línguas envolvidos neste estudo, apesar de se realizarem em um único gênero, concretizam-se de maneira muito particular, em determinados contextos, o que evidencia a necessidade e a importância de se estudarem e se compararem, pontualmente, suas realizações textuais mais particulares.

\section{Discussão}

O trabalho prático da tradução, e até mesmo sua teoria, têm ensinado que muito dificilmente temos respostas, fórmulas e "truques" para resolver os impasses de tradução.

Sabemos que, no estudo e na realização de traduções, estamos em áreas em que não há muitas regras e em que cada texto abordado apresenta um desafio diferente, com suas particularidades e contextos. O que vai prevalecer, para uma boa 
tradução, será, além da experiência e conhecimento dos pares de idiomas envolvidos, a disposição do tradutor de estar atento às diferenças e de buscar soluções para elas, além do conhecimento do profissional a respeito do gênero que traduz, como é colocado na epígrafe deste texto.

Considerando o nivel de internacionalização e de alcance dos artigos científicos, toma-se como pressuposto que é desejável que seus textos não carreguem as marcas da língua de partida, para que sejam bem entendidos pelos seus leitores. De acordo com Azenha (1999), “(...) do tradutor técnico se exige, além do domínio de um saber específico, a habilidade de coordenar ações e garantir (...) a adequação do texto traduzido às condições de recepção", se esse for o objetivo da tradução. Esse foi um dos objetivos desta comparação, mostrar como uma expressão pode ser mais bem adaptada ao evento de recepção do texto, sem que o texto de partida - no caso, o português - seja "decalcado" na tradução para a língua estrangeira.

As unidades que levantamos com base nos corpora não dependem da área a que os textos selecionados pertencem, mas sim de certas convenções e regularidades próprias da retórica e dos modos de dizer nas duas línguas em questão, de um gênero específico. No caso do artigo científico, podemos dizer que alguns dos objetivos de uma boa retórica seriam dar credibilidade às proposições e convencer o leitor do que está sendo apresentado, além de apresentar e organizar o texto de forma coerente e coesa.

Os meios retóricos de que um autor se vale para fazer um texto acabam estando relacionados e sendo restringidos por valores, sistemas de crenças e práticas comuns à comunidade discursiva que receberá e /ou avaliará o texto. Em textos acadêmicos, as comunidades discursivas que mais restringem os autores são aquelas para as quais os textos estão sendo escritos e a da cultura de escrita na qual o autor foi socializado quando aprendeu a escrever, que é, em geral, sua cultura nacional (Mauranen 1993, p. 5). Essas duas culturas entram em questão e conflito em um trabalho de tradução, como não poderia deixar de ser. É tarefa do tradutor avaliar se (e como) acontecerá um processo de adaptação às formas retóricas da comunidade discursiva do texto de chegada. 
A Retórica Contrastiva, nos trabalhos de Enkvist (1991 e 1995), Nord (1991), Baker (1992) e Neubert-Shreve (1992), por exemplo, já demonstrou resultados interessantes, que tiveram contribuições para a tradução, com base nos pressupostos que a sustentam: (i) a linguagem e a escrita são fenômenos culturais; (ii) cada língua tem suas convenções retóricas e (iii) essas convenções retóricas e lingüísticas da língua materna interferem na escrita da língua de chegada. (Sáez, 2003)

Isto posto, convém aproximarmos deste trabalho uma reflexão a respeito do texto especializado, a linguagem de especialidade na situação real de comunicação (Maciel, 2001). Até então, deixamos que a abordagem de gênero predominasse neste trabalho; porém, é inegável que, ao tratarmos do gênero artigo científico, estamos falando inevitavelmente de sua materialização, o texto especializado, uma vez que os textos de artigos científicos, em geral, são resultado de um exercício de observação ou de uma atividade científica ou técnica em uma dada área.

De acordo com Hoffmann (2004), o texto especializado “(...) é instrumento e ao mesmo tempo resultado da atividade comunicativa exercida em relação a uma atividade especializada". Esse texto se realiza de maneiras específicas, determinadas pelas suas funções e pelo seu âmbito (Möhn \& Pelka, 1984 apud Hoffmann, 1994), ou, acrescentariamos, pelos critérios textuais e discursivos e pelos critérios temáticos. Portanto, um texto especializado não se define apenas pelo critério tema (área científica ou técnica), mas por “(...) particularidades de sua macroestrutura (articulação), por relações de coerência entre seus elementos e pela utilização de unidades sintáticas, lexicais, morfológicas e gráficofonéticas". As preferências para o uso dessas particularidades se condicionam às circunstâncias da interação verbal dos atores do evento comunicativo, dependendo, portanto, das peculiaridades do destinador e do destinatário (Maciel, 2001). Como se vê, muito do que se discute sobre gênero se sobrepõe a esses comentários.

Se tomamos as definições de gênero de Bakhtin, para quem cada esfera de atividade desenvolve tipos relativamente estáveis de enunciados - os gêneros -, e de Swales, para quem um gênero é uma classe de eventos comunicativos cujos membros com- 
partilham um conjunto de propósitos comunicativos; e, ainda, a definição de texto especializado de Hoffmann (2004), segundo o qual “(...) o texto especializado é instrumento e, ao mesmo tempo, resultado da atividade comunicativa exercida em relação a uma atividade especializada sócio-produtiva", perceberemos que elas têm pontos em comum, principalmente no que concerne ao gênero ser determinado por uma atividade específica, envolvendo membros em sociedade, que compartilham atividades comunicativas.

Dessa forma, por exemplo, a escolha que fazemos dos itens que servirão aos nossos propósitos nas circunstâncias em que se realiza a comunicação é determinada pela nossa "esfera de atividade" e pela comunidade da qual estamos falando. Obviamente, surgem também as questões de estilo; mas, como propõe Bakhtin, também o estilo estará condicionado ao meio em que se realiza o texto.

O que precisamos deixar claro é que o enfoque do texto por parte dessas duas áreas - estudos de gênero e estudos de texto especializado - são diferentes. Quando falamos em um gênero, estamos falando do geral, de um agrupamento de eventos comunicativos (entre esses, o texto) que se assemelham. Por outro lado, um texto especializado é o particular, é a matéria que compõe os textos que pertencem a um gênero.

Já mencionamos anteriormente que o reconhecimento dos gêneros, por parte dos tradutores, é essencial para que se conheçam melhor os padrões de gêneros e subgêneros, no intuito de entender seu funcionamento nas diferentes linguas e produzir textos condizentes com esses padrões nas línguas envolvidas. $\mathrm{O}$ estudo do texto de especialidade, assim como da terminologia que o compõe, também pode trazer resultados importantes para o estudo da tradução de textos especializados, principalmente quando ambos, texto especializado e terminologia, forem abordados através de uma perspectiva ampla, de inclusão e descritiva.

Cabré (1999) sustenta que o tradutor de textos especializados deve conhecer alguns parâmetros para que sua tradução consiga atingir os índices mínimos de qualidade, ou seja, além de ser verídica, do ponto de vista do conteúdo, e correta, do ponto de vista gramatical, deve ser adequada e soar natural. $\mathrm{O}$ 
"soar natural" inclui as preocupações que norteiam a pesquisa realizada e descrita aqui, em que mencionamos a Retórica Contrastiva, preocupada principalmente com a naturalidade do texto de chegada.

O que queremos também demonstrar é que a tradução precisa de uma Terminologia que considere o funcionamento da linguagem com toda a sua complexidade e que, assim, possa descrever, em melhores condições, "(...) a especificidade dos contextos discursivos nos quais os termos se manifestam em toda a sua diversidade de configurações" (Krieger, 2001: 37). Conforme esclarece ainda Krieger (loc. cit., p. 37), “(...) para poder dar conta dessa diversidade, os estudos de Terminologia não podem ficar restritos aos limites da frase, nem à formalidade da análise de seus componentes morfossintáticos". Acreditamos que o estudo realizado, enfocando elementos que estão no entorno dos termos, contribui para esse olhar mais amplo em direção ao texto técnico-científico.

Um pouco em oposição à informação de que a "Terminologia teórica, à medida que evolui, está cada vez mais reconhecendo a necessidade de que o texto especializado integre seus objetos de estudo" (Krieger \& Finatto, 2004), entendemos que o texto de especialidade não se tornaria objeto de estudo, mas uma condição sine qua non para um olhar sobre termos e fraseologias. Nesse ponto, estamos em conformidade com a idéia de que o texto “(...) deixa de ser um mero conjunto de 'termos técnicos' relacionados, tornando-se um todo de estudo e análise também para a Terminologia" (loc. cit.), porém, ressaltamos, não como objeto, mas como potencial ponto de partida para observações acerca das unidades lexicais terminológicas. Assim, por exemplo, um estudo mais dedicado poderia observar que algumas das expressões que descrevemos neste trabalho contribuem para delimitar sentidos e restringir interpretações nos textos dos artigos, tais como "no sentido de que", "no que diz respeito a", "de acordo $\operatorname{com} x$ ". Um olhar sobre o texto parece deixar claro que o rigor e a univocidade que a ciência necessita não são dados exclusivamente pelos termos, como parece ser o entendimento geral, mas pelo todo do texto, pela maneira como os termos são colocados em ação e também por outros fatores externos. 


\section{Considerações finais}

Buscamos mostrar, ao longo deste artigo, uma visão geral da metodologia empregada e dos principais pontos desenvolvidos e levantados na pesquisa de mestrado intitulada Marcadores textuais do artigo científico em comparação português e inglês - um estudo sob a perspectiva da tradução. Com o sentido de não tornar o texto deste artigo extenso demais, procuramos apresentar apenas os principais resultados alcançados e as observações a respeito dos marcadores textuais em estudo, bem como uma reflexão sobre como entendemos que áreas como Terminologia, Lingüística de Corpus e Tradução vêm-se entrelaçando com bons resultados.

Queremos ressaltar que entendemos que a Terminologia sempre se beneficiará com o desenvolvimento de estudos interdisciplinares, incluindo cada vez mais em seu olhar outros elementos da trama textual no estudo de seus objetos, para além do termo, como os adjetivos, os conectores, a diagramação do texto, a pontuação, a retórica, dentre outros. Os produtos aos quais se presta a Terminologia aplicada, ou seja, principalmente os dicionários e os glossários, ficam mais enriquecidos se observarem os entornos dos termos e o seu contexto de realização. A descrição sistemática do texto desenvolvida no âmbito da Terminologia pode auxiliar a percepção de peculiaridades textuais de determinadas áreas de conhecimento, o que pode, por exemplo, ser muito útil para o tradutor (Bevilacqua, 1998).

Entendemos também que a investigação que desenvolvemos sobre os marcadores textuais pode vir a contribuir para estudos voltados para o processamento da linguagem natural e para a tradução automática, em que se buscam regularidades de composição lingüística e textual com o intuito de "alimentar" programas e sistemas que tenham como meio ou fim o uso da linguagem. Essa é uma perspectiva futura de trabalho e continuação da investigação aqui iniciada, em que pretendemos ampliar o corpus para outras áreas de conhecimento (Medicina e Química, em parceria com o Projeto TextQuim-UFRGS) e estudar como é possivel extrair automaticamente dos textos informações relevantes para o ato tradutório, tais como os marcadores textuais aqui apresentados. 


\section{Referências bibliográficas}

AZENHA Jr., J. (1999) Tradução técnica e condicionantes culturais primeiros passos para um estudo integrado. São Paulo: Humanitas/USP.

BAKER, M. (1992) In other words. In: A coursebook on translation. London: Routledge.

BAKHTIN, M. M. (1997) Estética da criação verbal. $2^{\mathrm{a}}$ ed. São Paulo: Martins Fontes.

BERBERSARDINHA, T. (2004) Lingüística de Corpus. Barueri: Manole. BENEDETTI, I. C. Prefácio. In: BENEDETTI, I. \& SOBRAL, A. (orgs.) (2003) Conversas com tradutores - balanços e perspectivas da tradução. São Paulo: Editora Parábola.

BEVILACQUA, C. (1998) Unidades fraseológicas especializadas: novas perspectivas para sua tradução e tratamento. v. 12, n. 26, p. 119132. [Porto Alegre]: Organon.

CABRÉ, M. T. (1999) La terminología: representación y comunicación. Barcelona: Institut Universitari de Linguística Aplicada.

ENKVIST, N. E. Discourse type, text-type and cross-cultural rhetoric. In: TIRKKONEN-CONDIT, S. (ed.). (1991) Empirical research in translation and intercultural studies. Tübingen: Gunter Narr Verlag. p. 5-16.

. Discourse organization, crosscultural rhetoric and translation. In: WARVIK, B.; TANSKANEN, S.-K. \& HILTUNEN, R. (eds.). (1995) Organization in discourse. Proceedings from the Turku Conference, Anglicana Turkuensia $\mathrm{N}^{\circ}$ 14. Turku: University of Turku. p. 41-58. HALLIDAY, M. A. K. (1985) An introduction to functional grammar. London: Edward Arnold.

HURTADO ALBIR, A. (2001) Traducción y traductología. Introducción a la traductologia. Madrid: Ediciones Cátedra.

KRIEGER, M._G. A face lingüística da terminologia. In: KRIEGER, M.G. \& MACIEL, A.M.B. (orgs.). (2001a) Temas de terminologia. Porto Alegre/São Paulo: Universidade Federal do Rio Grande do Sul/Humanitas/Universidade de São Paulo.

; FINATTO, M. J. B. (2004) Introdução à terminologia. Teoria e prática. São Paulo: Contexto.

MACIEL, A. M. B. (2001) Para o reconhecimento da especificidade do termo jurídico [tese de doutorado]. Porto Alegre: UFRGS. 299 p. 
MAURANEN, A. (1993) Contrastive ESP rhetoric: metatext in FinnishEnglish economics texts. English for Specific Purposes, v. 12, p. 3-22.

NEUBERT, A. \& SHREVE, G. (1992) Translation as text. Kent: The Kent State University Press.

NORD, C. (1991) Text analysis in translation. Amsterdam: Rodopi.

SÁEZ, F. T. Culture in writing: discourse markers in English and Spanish student writing. (2003) In: Departamento de Didáctica de la Lengua y la Literatura (eds.). Tadea seu liber de Amicitia. Granada: Imprenta Generalife. p. 345-364. Disponivel em: http://www.ugr.es/ ftsaez/ tadea.pdf.

SWALES, J. M. (1990) Genre analysis. English in academic and research settings. Cambridge: Cambridge University Press. 